\title{
Increasing Quantum Efficiency of Polymer Solar Cells with Efficient Exciton Splitting and Long Carrier Lifetime by Molecular Doping at Heterojunctions
}

Han Yan, ${ }^{*} \uparrow$ Yabing Tang, ${ }^{\dagger}$ Xinyu Sui, ${ }^{\ddagger}$ Yucheng Liu, ${ }^{\S}$ Bowei Gao, $״$ Xinfeng Liu, Shengzhong(Frank) Liu, ${ }^{\S}$ Jianhui Hou," and Wei Ma* ${ }^{*}$

$\dagger$ State Key Laboratory for Mechanical Behavior of Materials, Xi' an Jiaotong University, Xi' an 710049, P. R. China

$\$$ Division of Nanophotonics; CAS Key Laboratory of Standardization and Measurement for Nanotechnology; CAS Center for Excellence in Nanoscience; National Center for Nanoscience and Technology, Beijing 100191, P. R. China

$\S$ Key Laboratory of Applied Surface and Colloid Chemistry, National Ministry of Education; Shaanxi Key Laboratory for Advanced Energy Devices; Shaanxi Engineering Lab for Advanced Energy Technology; Institute for Advanced Energy Materials; School of Materials Science and Engineering, Shaanxi Normal University, Xi'an 710119, P. R. China

"State Key Laboratory of Polymer Physics and Chemistry; Beijing National Laboratory for Molecular Sciences; CAS Research/Education Center for Excellence in Molecular Sciences; Institute of Chemistry, Chinese Academy of Sciences, Beijing 100049, P. R. China

\section{Corresponding Author}

*E-mail: mseyanhan@xjtu.edu.cn (H. Yan.); msewma@xjtu.edu.cn (W. Ma) 


\section{Experimental Section}

Materials: PBDB-T and ITIC were purchased from Solarmer Materials Inc. BCF, zinc acetate dihydrate, ethanolamine, and 2-methoxyethanol were purchased from Sigma-Aldrich. All chemicals in the experiments were used as received.

Instrumentation: The $\mathrm{J}-\mathrm{V}$ curves were measured in the $\mathrm{N}_{2}$ glovebox under AM $1.5 \mathrm{G}$ (100 mW/cm²) using an AAA solar simulator (SS-F5-3A, Enli Technology Co., Ltd.) calibrated with a standard photovoltaic cell equipped with KG5 filter and a Keithley 2400 source meter. Typical cells have device areas of $4 \mathrm{~mm}^{2}$, defined by a mask with aperture aligned with the device area. The EQE was measured by Solar Cell Spectral Response Measurement System QE-R3018 (Enli Technology Co., Ltd.). The light intensity was calibrated with a standard Si photovoltaic cell. The absorption spectra were acquired on a Shimadzu UV-3600 Plus. The thickness of active layer was measured by Bruker Dektak XT. Femtosecond transient absorption measurements were carried out with a temporal resolution of $\sim 120 \mathrm{fs} t$ on a commercial femtosecond TAS system (HELIOS, Ultrafast Systems). Briefly, the fundamental $800 \mathrm{~nm}$ pulse from a regenerative amplifier ( $80 \mathrm{fs}, 1 \mathrm{kHz}, 2.5 \mathrm{~mJ}$ per pulse, Coherent, Astrella), seeded by a oscillator ( $35 \mathrm{fs}, 80 \mathrm{MHz}$, Coherent Vitara-S ), was used to pump an optical parametric amplifier (Coherent, OperA Solo) to generate excitation pulse peaking at $400 \mathrm{~nm}$. The pump beam was chopped by $500 \mathrm{~Hz}$ in order to get the shot to shot measurement. The pump fluence was attenuated to $\sim 10 \mu \mathrm{J} \mathrm{cm}^{-2}$ with two variable neutral density filter. The probe light was get where a small fraction of the $800 \mathrm{~nm}$ output from the Astrella was fed to a sapphire crystal in the HELIOS for 
generating the white light continuum (WLC). Steady and time-resolved PL measurements were taken using a PicoQuant FT-300 and FT-100 with different excitation wavelength. UPS spectrum was collected on ESCALAB Xi+ (Thermo Fisher). The TPV measurements were performed by the all-in-one characterization platform Paios developed and commercialized by Fluxim AG, Switzerland Device Fabrication: The invertd PSCs were fabricated with a configuration of ITO/ZnO/Active Layer/MoO$/ \mathrm{Ag}$. The normal PSCs were fabricated with a configuration of ITO/PEDOT:PSS/Active Layer/Ca/Al. The ITO substrates were cleaned by sequential sonication in deionized water, acetone and isopropanol for 20 min of each step. After UVO treatment for $20 \mathrm{~min}$, and electron-transporting layer of $\mathrm{ZnO}$ was deposited by spin-coating a $\mathrm{ZnO}$ precursor solution (dissolving zinc acetate in 2-methoxyethanol with ethanolamine) at $4500 \mathrm{rpm}$ for $40 \mathrm{~s}$, followed by thermal annealing at $200{ }^{\circ} \mathrm{C}$ for $30 \mathrm{~min}$. The hole-transporting layer of PEDOT:PSS was deposited by spin-coating at $4500 \mathrm{rpm}$ for $40 \mathrm{~s}$, followed by thermal annealing at 150 ${ }^{\circ} \mathrm{C}$ for 10 min. PHJ devices were fabricated by floating- film-transfer method, which was used to stack the PBDB-T layer on the ITIC layer. The PBDB-T film was floated in water, and then it was scooped up by ITIC coated cathode substrate. BHJ devices were fabricated by spin-coating PBDB-T/ITIC blend solution or sequentially spin-coating each single component with different processing conditions in the $\mathrm{N}_{2}$ glove box. Finally, $10 \mathrm{~nm} \mathrm{MoO}_{3} / 100 \mathrm{~nm} \mathrm{Ag}$ or $20 \mathrm{~nm} \mathrm{Ca} / 80 \mathrm{~nm} \mathrm{Al} \mathrm{were}$ sequentially deposited as the anode or cathode at a vacuum level of $<1 \times 10^{-4} \mathrm{~Pa}$. 
GIWAXS characterization: GIWAXS measurements were performed at beamline 7.3.3 at the Advanced Light Source (ALS). Samples were prepared on Si substrates using identical blend solutions as those used in devices. The $10 \mathrm{keV}$ X-ray beam was incident at a grazing angle of $0.12-0.16^{\circ}$, selected to maximize the scattering intensity from the samples. The scattered X-ray were detected using a Dectris Pilatus 2M photon counting detector.

\section{TAS lifetime:}

The TAS decay signals are fitted using a Gaussian response function convoluted with multi-exponential decay function:

$$
\mathrm{e}^{-\frac{\mathrm{t}^{2}}{\tau_{\text {pulse }}^{2}}} \otimes \sum A_{i} e^{-\frac{t}{\tau_{i}}}
$$

and the TAS lifetime is further calculated according to the following equation:

$$
\tau=\frac{\sum A_{i} \tau_{i}^{2}}{\sum A_{i} \tau_{i}}
$$

\section{Carrier lifetime calculation:}

The carrier lifetime is calculated according to the TPV tests. In organic photovoltaics, the $\mathrm{V}_{\text {oc }}$ can be calculated by the following equation:

$$
\mathrm{V}_{\mathrm{oc}}=\frac{\mathrm{E}_{\mathrm{g}}}{\mathrm{q}}-\frac{\mathrm{n}_{\mathrm{eff}} \mathrm{k}_{\mathrm{B}} \mathrm{T}}{\mathrm{q}} \ln \left(\frac{\mathrm{N}^{2}}{\mathrm{np}}\right)
$$

where $\mathrm{E}_{\mathrm{g}}$ is the bandgap of organic photovoltaics, $\mathrm{n}_{\mathrm{eff}}$ is the effective ideality factor, $\mathrm{kB}_{\mathrm{B}}$ is the Boltzmann's constant, $\mathrm{T}$ is the absolute temperature, $\mathrm{q}$ is the elemental charge, and $\mathrm{n} \& \mathrm{p}$ are the density of electrons and holes.

The $\mathrm{V}_{\mathrm{oc}}$ decay is calculated by deriving the upper equation: 


$$
\mathrm{q} \frac{\mathrm{dV} \text { oc }}{d t}=k_{B} T \frac{d \ln (n p)}{d t}
$$

Then the carrier lifetime is calculated from the TPV data:

$$
\tau=-\frac{\mathrm{k}_{\mathrm{B}} \mathrm{T}}{\mathrm{q}}\left(\frac{\mathrm{dV}_{\mathrm{oc}}}{\mathrm{dt}}\right)^{-1}
$$

We have added these details in the supporting information. 

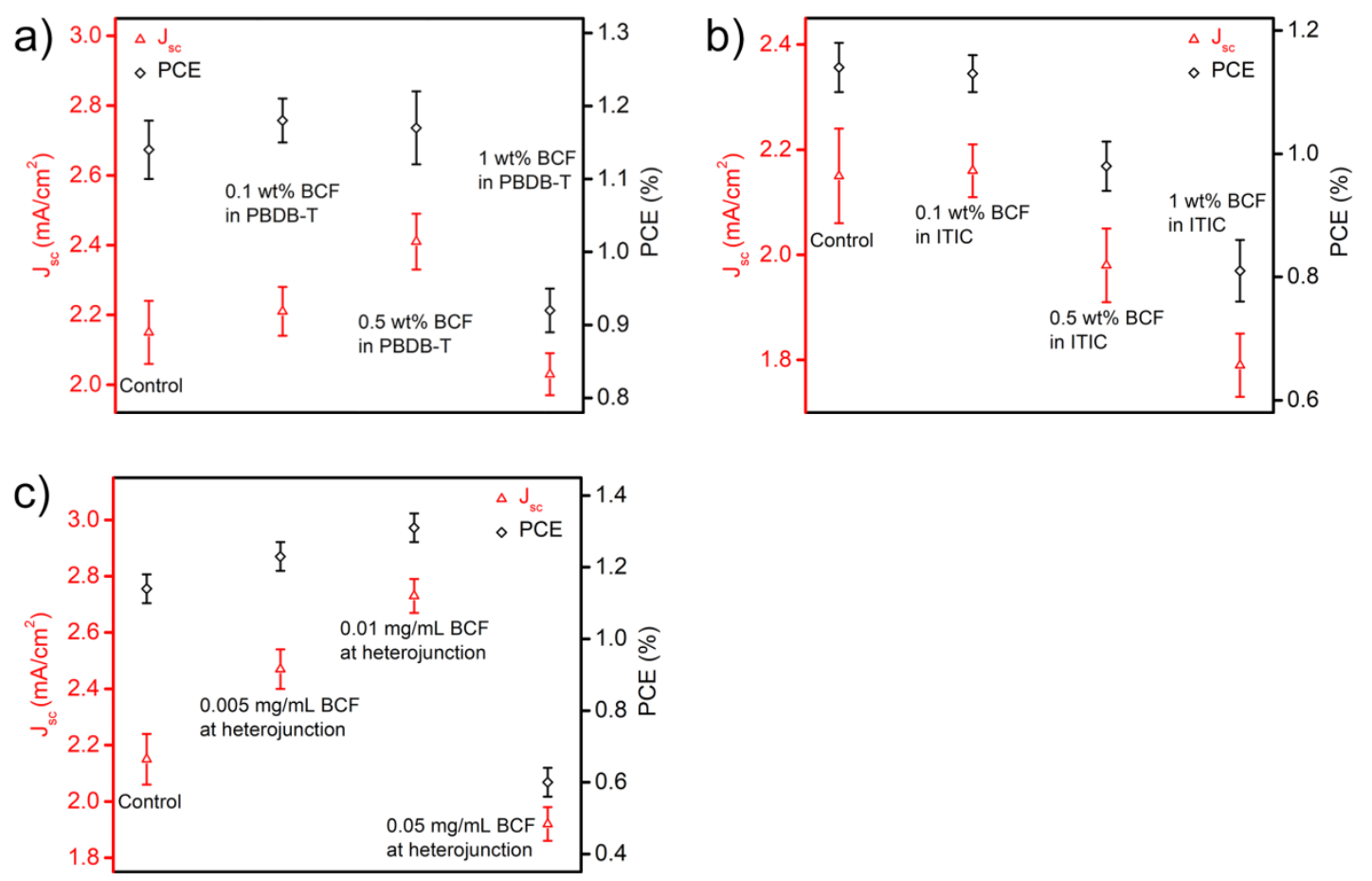

Figure S1. Plots of $\mathrm{J}_{\mathrm{sc}}$ and PCE in PHJ device structure in accordance with Table 1. 

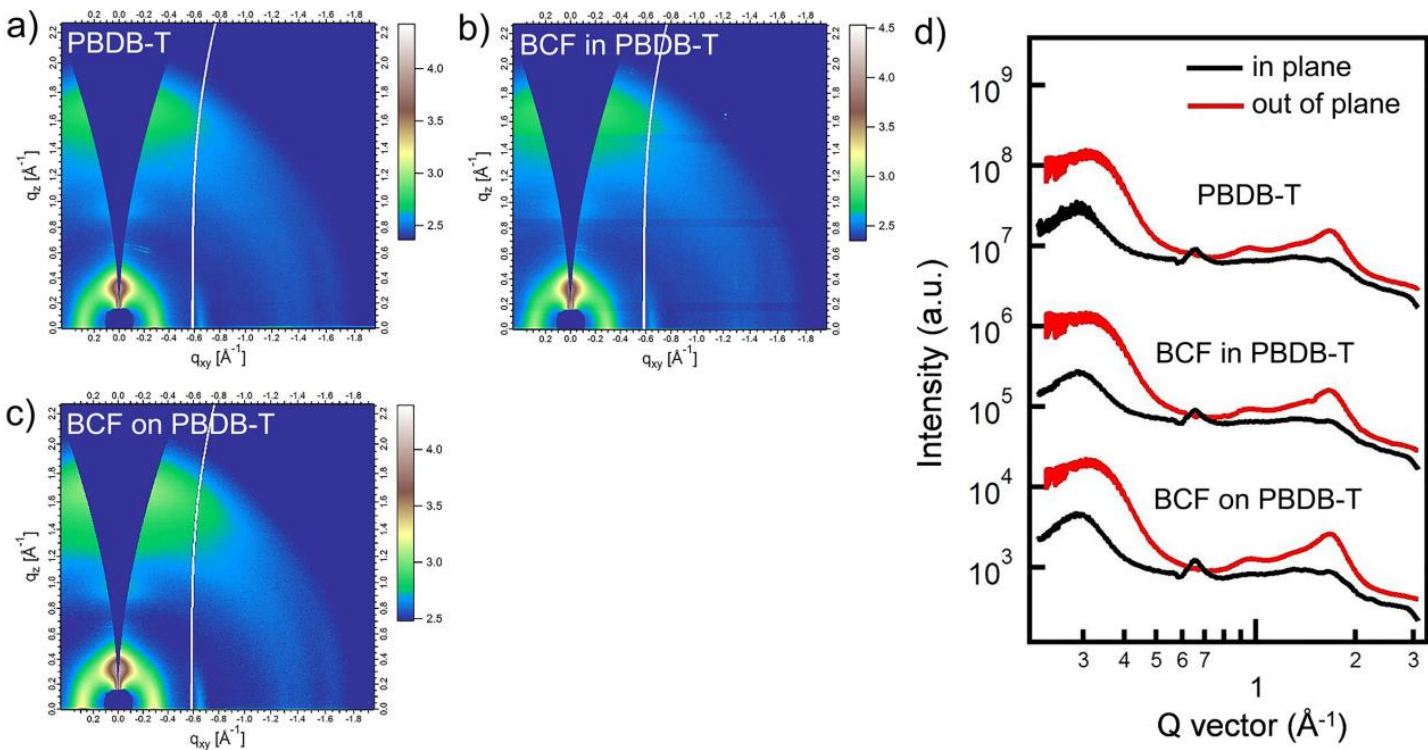

Figure S2. a-c) GIWAXS patterns of PBDB-T films: a) neat; b) $0.1 \mathrm{wt} \% \mathrm{BCF}$ in PBDB-T; c) $0.01 \mathrm{mg} / \mathrm{mL}$ BCF on PBDB-T. d) Corresponding in plane and out of plane line cuts.

Table S1. The fitted peak position and coherence length from GIWAXS patterns for PBDB-T films.

\begin{tabular}{|c|c|c|c|c|}
\hline Materials & Location $\left(\AA^{-1}\right)$ & d-spacing ( $\mathbf{A}^{-1}$ & FWHM $\left(\AA^{-1}\right)$ & CL $(\mathbf{n m})$ \\
\hline PBDB-T & 0.29 & 21.66 & 0.086 & 6.55 \\
\hline BCF in PBDB-T & 0.29 & 21.58 & 0.092 & 6.15 \\
\hline BCF on PBDB-T & 0.29 & 21.64 & 0.093 & 6.11 \\
\hline
\end{tabular}



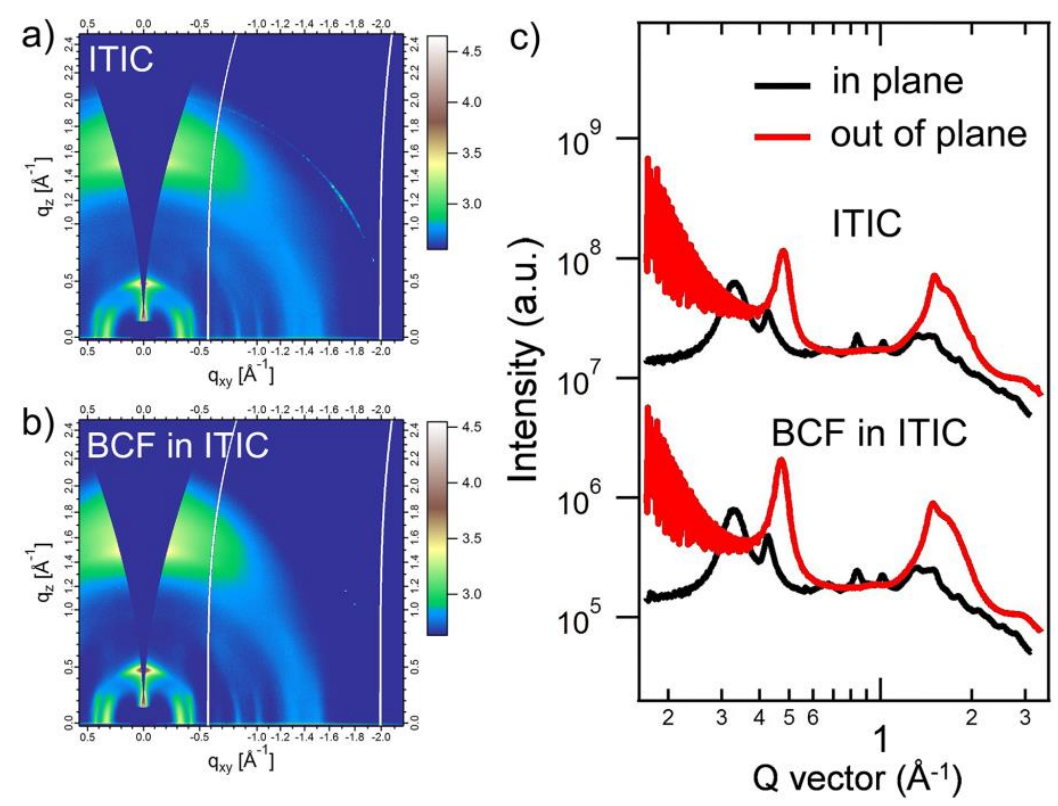

Figure S3. a, b) GIWAXS patterns of ITIC films: a) neat; b) $0.1 \mathrm{wt} \%$ BCF in ITIC. c) Corresponding in plane and out of plane line cuts.

Table S2. The fitted peak position and coherence length from GIWAXS patterns for ITIC films.

\begin{tabular}{|c|c|c|c|c|}
\hline Materials & Location $\left(\AA^{-1}\right)$ & d-spacing $(\AA)$ & FWHM $\left(\AA^{-1}\right)$ & CL $(\mathbf{n m})$ \\
\hline ITIC & 0.33 & 19.12 & 0.063 & 8.92 \\
\hline BCF in ITIC & 0.33 & 19.11 & 0.064 & 8.83 \\
\hline
\end{tabular}




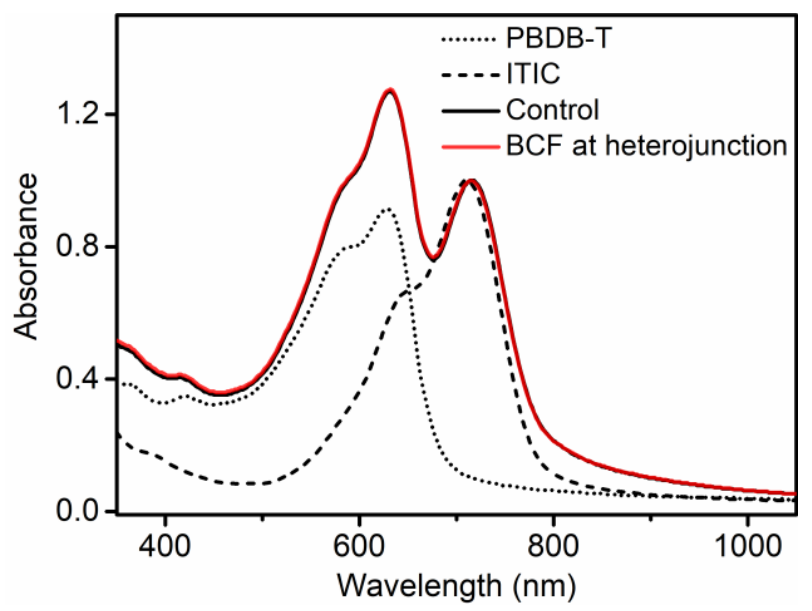

Figure S4. Steady absorption spectra of neat and PHJ films.
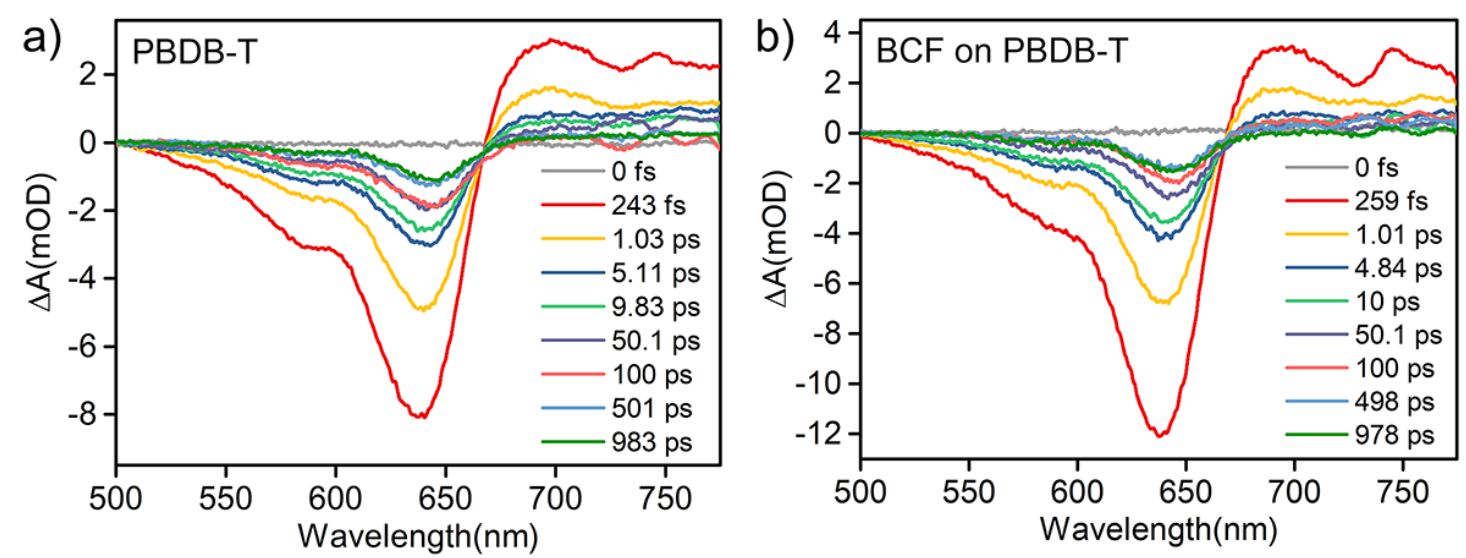

Figure S5. Transient absorption spectra of PBDB-T films using $400 \mathrm{~nm}$ laser pulse excitation: a) neat; b) BCF on PBDB-T.

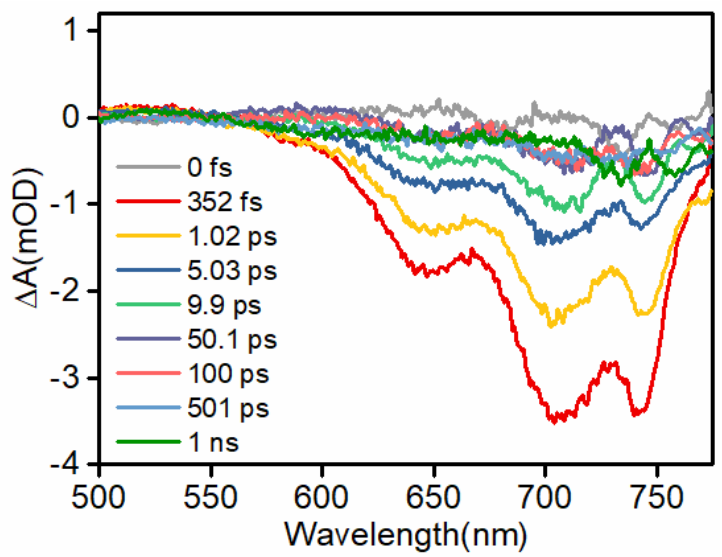

Figure S6. Transient absorption spectra of ITIC film using $400 \mathrm{~nm}$ laser pulse excitation. 

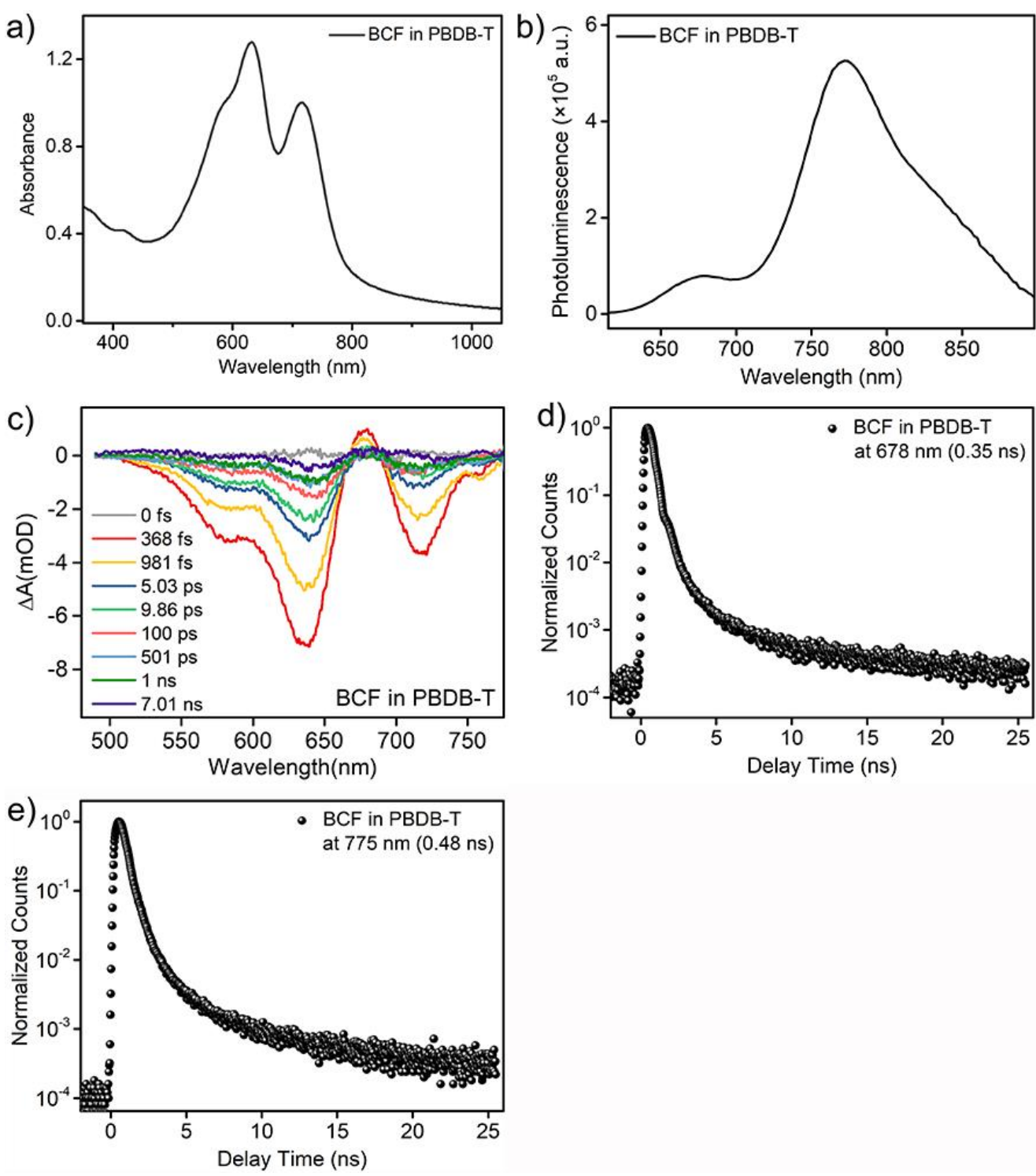

Figure S7. Spectral studies of $0.1 \mathrm{wt} \%$ BCF in PBDB-T: a) steady absorption spectra;

b) steady state photoluminescence spectra recorded using $400 \mathrm{~nm}$ excitation; c) transient absorption spectra using $400 \mathrm{~nm}$ laser pulse excitation; $\mathrm{d}$ and e) transient PL spectra probing the peak at $678 \mathrm{~nm}$ and $775 \mathrm{~nm}$. 

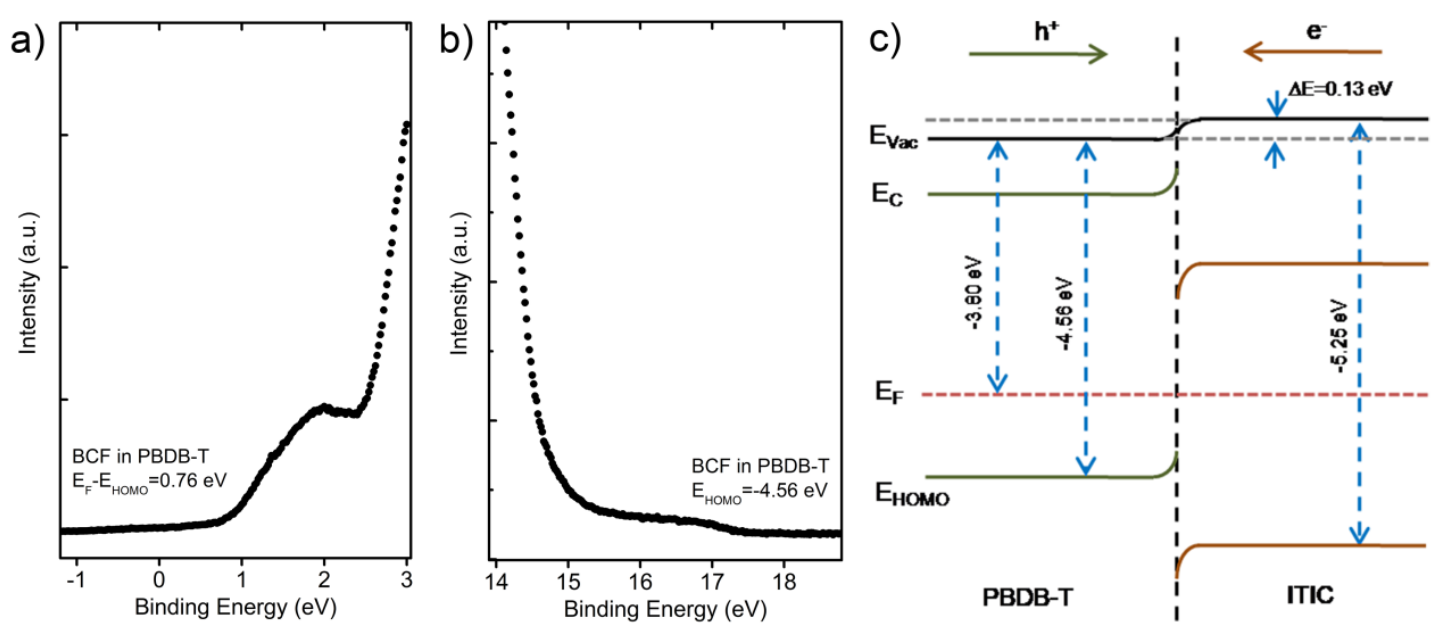

Figure S8. UPS spectra of the $0.1 \mathrm{wt} \%$ BCF in PBDB-T film under $-10 \mathrm{~V}$ bias: a) low and b) high energy part of PBDB-T films. c) The scheme of band diagrams according to the UPS spectra.
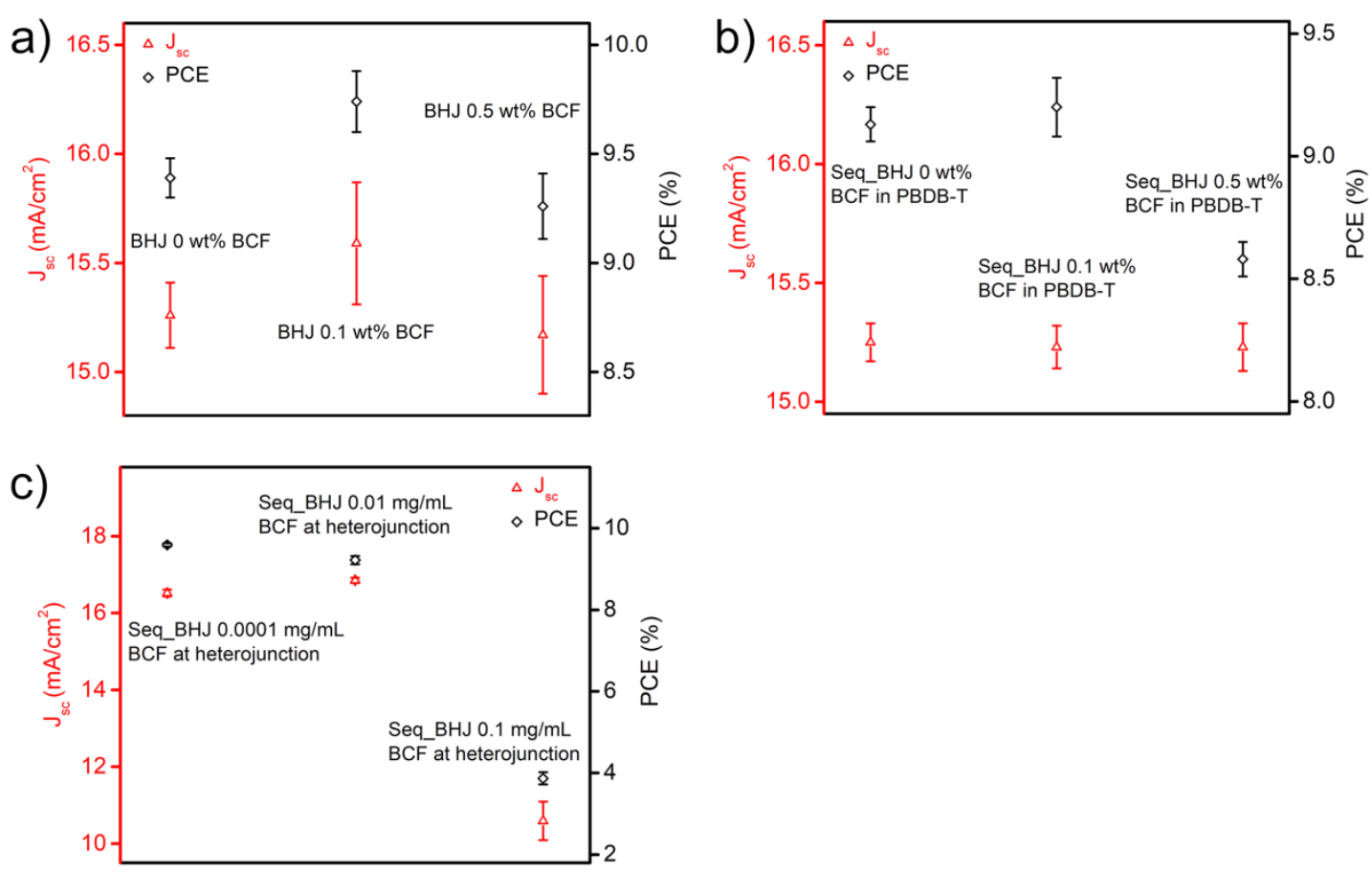

Figure S9. Plots of $\mathrm{J}_{\mathrm{sc}}$ and PCE under various fabricating processes in accordance with Table 2. 

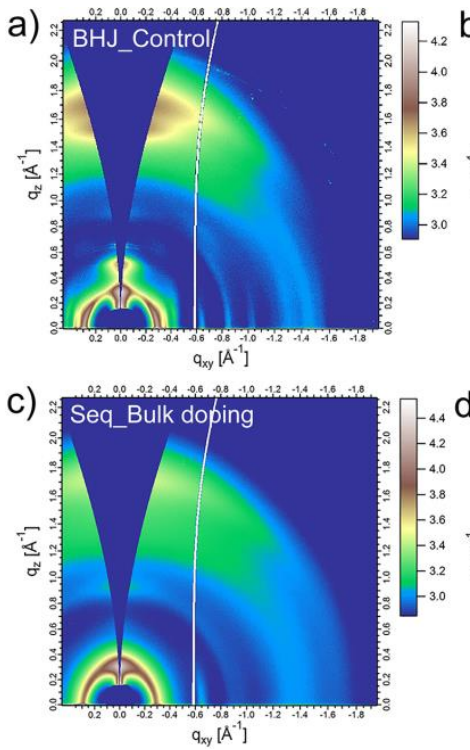
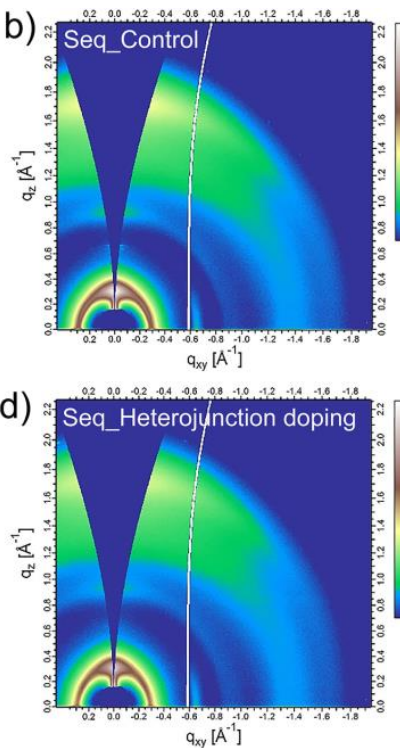

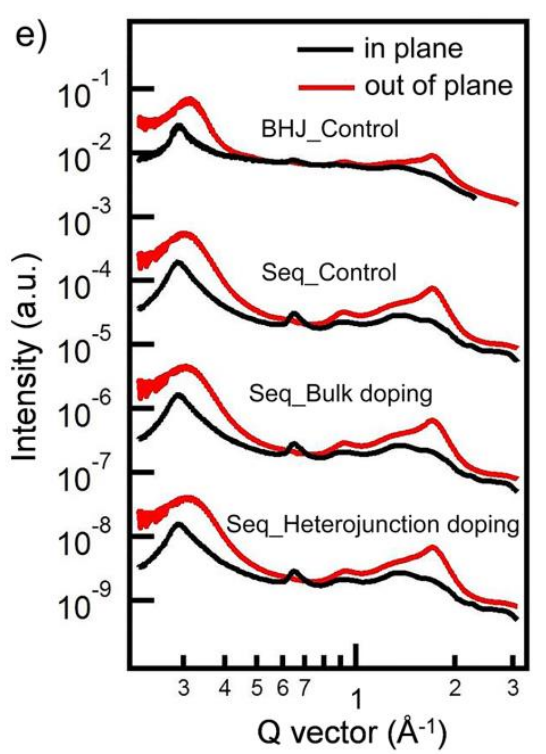

Figure S10. a-c) GIWAXS patterns of BHJ films: a) conventional BHJ; b) sequential spin-coating BHJ; c) sequential spin-coating $0.1 \mathrm{wt} \%$ BCF doped BHJ; d) sequential spin-coating $0.01 \mathrm{mg} / \mathrm{mL}$ BCF interfacial doped BHJ. e) Corresponding in plane and out of plane line cuts.

Table S3. The fitted peak position and coherence length from GIWAXS for BHJ films.

\begin{tabular}{|c|c|c|c|c|}
\hline Materials & Location $\left(\AA^{-1}\right)$ & d-spacing $(\AA)$ & FWHM $\left(\AA^{-1}\right)$ & CL $(\mathbf{n m})$ \\
\hline BHJ_Control & 0.29 & 21.64 & 0.028 & 20.05 \\
\hline Seq_Control & 0.29 & 21.79 & 0.035 & 16.05 \\
\hline Seq_Bulkdoping & 0.29 & 21.62 & 0.042 & 13.55 \\
\hline $\begin{array}{c}\text { Seq_Heterojunction } \\
\text { doping }\end{array}$ & 0.29 & 21.59 & 0.040 & 14.00 \\
\hline
\end{tabular}

